\title{
Diagnostics of Anodic Stripping Mechanisms under Square-Wave Voltammetry Conditions Using Bismuth Film Substrates
}

\author{
Valentin Mirceski, ${ }^{\dagger, \S}$ Samo B. Hocevar, ${ }^{\ddagger}$ Bozidar Ogorevc, ${ }^{*}+$ Rubin Gulaboski, $^{\dagger, \S}$ and Ivan Drangov ${ }^{\dagger}$ \\ ${ }^{\dagger}$ Institute of Chemistry, Faculty of Natural Sciences and Mathematics, "Ss Cyril and Methodius" University, P.O. Box 162, 1000 \\ Skopje, Republic of Macedonia \\ ${ }^{\ddagger}$ Analytical Chemistry Laboratory, National Institute of Chemistry, Hajdrihova 19, SI-1000, Ljubljana, Slovenia \\ ${ }^{\S}$ Department of Chemistry, University Goce Delčev, Štip, Republic of Macedonia
}

ABSTRACT: A mechanistic study to provide diagnostics of anodic stripping electrode processes at bismuth-film electrodes is presented from both theoretical and experimental points of view. Theoretical models for three types of electrode mechanisms are developed under conditions of square-wave voltammetry, combining rigorous modeling based on integral equations and the step function method, resulting in derivation of a single numerical recurrent formula to predict the outcome of the voltammetric experiment. In the course of the deposition step, it has been assumed that a uniform film of the metal analyte is formed on the bismuth substrate, in situ deposited onto a glassy carbon electrode surface, without considering mass transfer within either the bismuth or

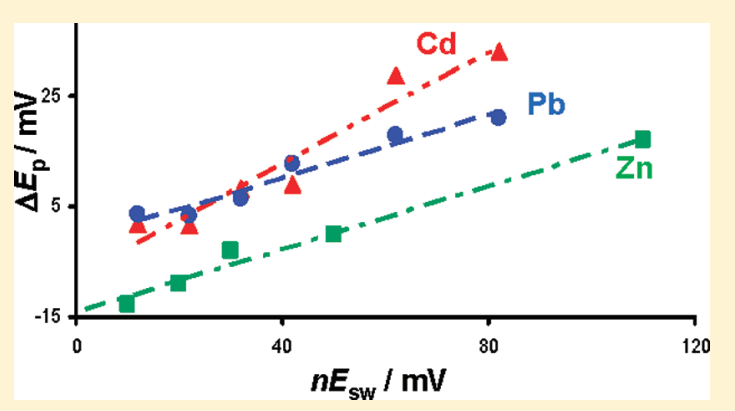
the metal analyte film. Theoretical data are analyzed in terms of dimensionless critical parameters related with electrode kinetics, mass transfer, adsorption equilibria, and possible lateral interactions within the deposited metal particles. Theoretical analysis enables definition of simple criteria for differentiation and characterization of electrode processes. Comparing theoretical and experimental data, anodic stripping processes of zinc(II), cadmium(II), and lead(II) are successfully characterized, revealing significant differences in their reaction pathways. The proposed easy-to-perform diagnostic route is considered to be of a general use while the bismuth film exploited in this study served as a convenient nonmercury model substrate surface.

\begin{abstract}
A nodic stripping voltammetry (ASV) is recognized as a powerful electroanalytical methodology in the trace metal analysis. It is attributed with a high sensitivity (down to $10^{-10}$ $\mathrm{M})$, capability for simultaneous determination of several metal analytes, and a relatively low cost of instrumentation that can be assembled also in a portable form and is readily miniaturized. Most frequently, ASV has been conducted using hanging mercury drop or mercury film electrodes. ${ }^{1}$ The use of mercury as an electrode material has been widely accepted over the past decades due to the extremely favorable signal-tobackground ratio, which is a consequence of the effective preconcentration step combined with advanced measurement procedures. However, the high toxicity of mercury, in combination with the inconvenience of its handling, is becoming the main drawback of mercury-based ASV. For this reason, various types of working electrodes, based on, for example, platinum, ${ }^{2}$ silver, ${ }^{3}$ or iridium ${ }^{4}$ have been proposed as an alternative to mercury. However, their performances for the trace metal analysis are rather unfavorable compared to the mercury counterpart. Recently, a significant step forward has been done by the introduction and development of bismuth ${ }^{5}$ and antimony-based electrodes. ${ }^{6-8}$

Bismuth film electrodes (BiFEs) consist of an electrochemically generated thin bismuth film, deposited predominantly on carbon-based substrates, such as glassy carbon, ${ }^{9}$ carbon fiber, ${ }^{10}$ or screen-printed carbon. ${ }^{11}$ The bismuth film is mechanically
\end{abstract}

stable, exhibiting high electroanalytical performance for trace metal analysis well comparable to the mercury electrodes. Besides measuring toxic metal ions with anodic stripping voltammetry, BiFEs have been successfully applied for adsorptive stripping voltammetric measurement of metal complexes, ${ }^{12-14}$ as well as for cathodic (stripping) voltammetric determination of several organic compounds. ${ }^{15}$ Taking into account that bismuth is an environmentally friendly electrode material with a very low toxicity, bismuth-based electrodes have become highly attractive and suitable electrodes for the on-site environmental monitoring and clinical testing of several toxic metals. In the past few years, the popularity of bismuth electrodes increased significantly, representing a frontier research in the sensor development area for trace metal analysis. ${ }^{16-18}$

Most of the studies published so far with BiFEs are of empirical nature, being oriented toward analytical applications of these electrodes. Thus, there is a strong necessity for fundamental research in the field devoted to mechanistic, kinetic, and thermodynamic aspects of electrode processes at the BiFEs. Moreover, there is a lack of a solid theoretical background, which can help in elucidation of the electrode

Received: January 13, 2012

Accepted: March 31, 2012

Published: March 31, 2012 
mechanisms and optimization of the response under voltammetric or chronoamperometric conditions. In the present study, an attempt is made to provide a theory for simulations of three different electrode mechanisms (reaction schemes I, II, and III) in order to predict the outcome of the anodic stripping voltammetric experiments.

$$
\begin{gathered}
\mathrm{M}(\mathrm{Bi})(\mathrm{s})=\mathrm{M}^{\mathrm{n}+}(\mathrm{aq})+n \mathrm{e}^{-} \\
\mathrm{M}(\mathrm{Bi})(\mathrm{s})=\mathrm{M}^{\mathrm{n}+}(\mathrm{ads})+n \mathrm{e}^{-} \quad \text { (II, no interactions) } \\
\uparrow \downarrow \beta \\
\mathrm{M}^{\mathrm{n}+}(\mathrm{aq}) \\
\mathrm{M}(\mathrm{Bi})(\mathrm{s})=\mathrm{M}^{\mathrm{n}+}(\mathrm{ads})+n \mathrm{e}^{-} \quad \text { (III, with interactions) } \\
\uparrow \downarrow \beta, a \\
\mathrm{M}^{\mathrm{n}+}(\mathrm{aq})
\end{gathered}
$$

In all mechanisms, it is assumed that, in the course of the deposition step, a solid metal analyte deposit $(\mathrm{M}(\mathrm{Bi})(\mathrm{s}))$ is formed on the surface of Bi-film substrate, without significant mass transfer within the Bi-film. The metal analyte film is assumed to be uniform with respect to the energy required for the anodic stripping process. The complexity that might arise in the experimental reality due to a multilayer formation and consequent thermodynamic differences between the first and upper metal analyte layers are not considered. This assumption is justified by the fact that, in the trace analysis of metal analytes such as zinc(II), cadmium(II), and lead(II) at BiFEs, the anodic stripping of each metal is most frequently associated with a single voltammetric peak..$^{9-11,16-18}$ This implies formation of a relatively uniform metal analyte film due to the capability of $\mathrm{Bi}$ to form alloys with these metals. All electrode mechanisms are analyzed under conditions of square-wave anodic stripping voltammetry (SWASV), as this technique is the most frequently applied for analytical application in combination with BiFEs and it is superior for kinetic measurements and for mechanistic studies. ${ }^{19}$ We note that the theoretical fundamentals of SWASV at mercury film electrodes have been established primarily by Osteryoung et al., ${ }^{20,21}$ Stojek et al., ${ }^{22}$ Lovric et al., ${ }^{23}$ and Compton et al. ${ }^{24,25}$

While Reaction I is considered as a simple anodic stripping mechanism, in Reaction II, it is assumed that the metal analyte ions $\left(\mathrm{M}^{\mathrm{n}+}(\mathrm{aq})\right)$ are involved in adsorption equilibrium at the electrode surface $\left(\mathrm{M}^{\mathrm{n}+}(\mathrm{ads})\right)$. There are two justifications for the mechanism II and the application of SWASV; i.e., metal deposition is frequently accompanied with the adsorption phenomena $^{26}$ and SWASV is particularly sensitive to the adsorption coupled electrode processes. ${ }^{19}$ To account for the inherent differences between the deposits of different metal analytes, in the mechanism III, interactions between the metal particles are considered, invoking the theory developed by Laviron for simple adsorbates. ${ }^{27}$ In the present study, some of the theoretical predictions are qualitatively correlated with square-wave anodic stripping voltammograms of zinc(II), cadmium(II), and lead(II) as model metal ions at the BiFE.

\section{THEORETICAL MODEL}

The mathematical model is formulated as follows:

$$
\begin{aligned}
& \frac{\partial c_{\mathrm{M}^{\mathrm{n}}}(x, t)}{\partial t}=D \frac{\partial^{2} c_{\mathrm{M}^{\mathrm{n}+}}(x, t)}{\partial x^{2}} \quad \text { for reactions I-III } \\
& t=0, x \geq 0: \quad c_{\mathrm{M}^{\mathrm{n}+}}(x, 0)=c_{\mathrm{M}^{\mathrm{n}+}}^{*} \quad \Gamma_{\mathrm{M}}(0)=0
\end{aligned}
$$

for reactions I-III

$\Gamma_{\mathrm{M}^{\mathrm{n}}}(0)=0 \quad$ for reactions II and III

$t>0, x \rightarrow \infty: \quad c_{\mathrm{M}^{\mathrm{n}+}} \rightarrow c_{\mathrm{M}^{\mathrm{n}+}}^{*} \quad$ for reactions I-III

$t>0, x=0: \quad \beta c_{\mathrm{M}^{\mathrm{n}}+}(0, t)=\Gamma_{\mathrm{M}^{\mathrm{n}}+}(t)$

for reactions II and III

$\left.D \frac{\partial c_{\mathrm{M}^{\mathrm{n}}}(x, t)}{\partial x}\right|_{x=0}=\frac{I(t)}{n F A} \quad$ for reaction $\mathrm{I}$

$\left.D \frac{\partial c_{\mathrm{M}^{\mathrm{n}}}(x, t)}{\partial x}\right|_{x=0}-\frac{\mathrm{d} \Gamma_{\mathrm{M}^{\mathrm{n}+}}(t)}{\mathrm{d} t}=\frac{I(t)}{n F A}$

for reactions II and III

$\frac{\mathrm{d} \Gamma_{\mathrm{M}}(t)}{\mathrm{d} t}=\frac{I(t)}{n F A} \quad$ for reactions I-III

Here, $c^{*} \mathrm{M}^{\mathrm{n}+}$ and $\Gamma_{\mathrm{M}^{\mathrm{n}+}}$ are bulk and surface concentrations of the metal analyte ion $\mathrm{M}^{\mathrm{n}+}(\mathrm{aq})$, respectively, whereas $\Gamma_{M}$ is the surface concentration of the metal analyte $\mathrm{M}(\mathrm{Bi})(\mathrm{s})$. In addition, $D$ is diffusion coefficient, $x$ is distance from the electrode surface, $t$ is time, $n$ is number of exchanged electrons, $F$ is Faraday constant, $A$ is electrode surface area, and $I$ is electric current. As at the beginning of the experiment, the initial reactant is present as an oxidized species; i.e., $\mathrm{M}^{\mathrm{n}+}(\mathrm{aq})$, the reductive current is defined with a positive sign. Equation 5 implies that a linear adsorption isotherm is assumed for the reacting metal ions with an adsorption constant $\beta$. At the electrode surface, the following kinetic equations apply:

$$
\frac{I(t)}{n F A}=k_{\mathrm{s}} \exp (-\alpha \phi)\left[c_{\mathrm{M}^{\mathrm{n}+}}(0, t)-\exp (\phi) r_{\mathrm{s}} \Gamma_{\mathrm{M}}(t)\right]
$$

for reaction $\mathrm{I}$

$$
\frac{I(t)}{n F A}=k_{\text {sur }} \exp (-\alpha \phi)\left[\Gamma_{M^{n+}}(t)-\exp (\phi) \Gamma_{M}(t)\right]
$$

for reaction II

$$
\begin{aligned}
& \frac{I(t)}{n F A}=k_{\text {sur }} \exp (-\alpha \phi)\left[\Gamma_{\mathrm{M}^{\mathrm{n}}}(t)-\exp (\phi) \exp (-2 a \theta)\right. \\
& \left.\Gamma_{\mathrm{M}}(t)\right] \quad \text { for reaction III }
\end{aligned}
$$

Here, $k_{\mathrm{s}}$ is heterogeneous standard rate constant in units of $\mathrm{cm}$ $\mathrm{s}^{-1}$ and $k_{\text {sur }}$ is the surface heterogeneous standard rate constant in units of $\mathrm{s}^{-1} \cdot r_{\mathrm{s}}=1 \mathrm{~cm}^{-1}$ is an auxiliary constant to account for the dimensional correctness of eq 9. In addition, $\alpha$ is the cathodic electron transfer coefficient, $\varphi=((n F) /(R T))\left(E-E_{\mathrm{c}}^{\ominus^{\prime}}\right)$ and is the dimensionless relative electrode potential defined versus the formal potential of each reaction, $a$ is the Frumkin interaction parameter, $\theta=\Gamma_{\mathrm{M}} / \Gamma_{\max }$ and is the fraction of the electrode covered by the metal analyte deposit, and $\Gamma_{\max }$ is the maximal surface concentration. $a$ is a dimensionless parameter, being positive for attractive and negative for repulsive forces. The kinetic eq 11 is a simplified form of the equation derived 
by Laviron for surface processes involving different types of interactions between immobilized redox active species. ${ }^{27}$ The solutions for the surface concentrations of electroactive species are

$$
c_{\mathrm{M}^{\mathrm{n}+}}(0, t)=c_{\mathrm{M}^{\mathrm{n}+}}^{*}-\int_{0}^{t} \frac{I(\tau)}{n F A \sqrt{D}} \frac{\mathrm{d} \tau}{\sqrt{\pi(t-\tau)}}
$$

for reaction I

$$
\begin{aligned}
& \Gamma_{\mathrm{M}^{\mathrm{n}+}}=\beta c_{\mathrm{M}^{\mathrm{n}+}}^{*}\left[1-\exp \left(a_{\mathrm{ads}}^{2} t\right) \operatorname{erfc}\left(a_{\mathrm{ads}} \sqrt{t}\right)\right] \\
& \quad-\int_{0}^{t} \frac{I(\tau)}{n F A} \exp \left[a_{\mathrm{ads}}{ }^{2}(t-\tau)\right] \operatorname{erfc}\left(a_{\mathrm{ads}} \sqrt{t-\tau}\right) \mathrm{d} \tau
\end{aligned}
$$

for reactions II and III

$$
\Gamma_{\mathrm{M}}=\int_{0}^{t} \frac{I(\tau)}{n F A} \mathrm{~d} \tau \quad \text { for reactions I-III }
$$

Here, $a_{\mathrm{ads}}=(D)^{1 / 2} / \beta$ and is an auxiliary adsorption parameter. Combining eqs 9-14 one obtains corresponding integral equations as general mathematical representations for reactions I-III, under voltammetric conditions. Numerical solutions, derived by the step function method ${ }^{28}$ and adopted for SWASV, are

$$
\Psi_{\mathrm{m}}=\frac{\lambda \mathrm{e}^{-\alpha \phi_{\mathrm{m}}}\left[1-\frac{2}{\sqrt{50 \pi}} \sum_{j=1}^{m-1} \Psi_{\mathrm{j}} S_{\mathrm{m}-\mathrm{j}+1}-\frac{\gamma \mathrm{e}^{\phi_{\mathrm{m}}}}{50} \sum_{j=1}^{m-1} \Psi_{\mathrm{j}}\right]}{1+\lambda \mathrm{e}^{-\alpha \phi_{\mathrm{m}}}\left(\frac{2}{\sqrt{50 \pi}}+\frac{\gamma \mathrm{e}^{\phi_{\mathrm{m}}}}{50}\right)}
$$

for reaction I

$$
\Psi_{\mathrm{m}}=\frac{\omega \mathrm{e}^{-\alpha \phi_{\mathrm{m}}}\left[\frac{1}{\rho}\left(1-\mathrm{e}^{\frac{\rho^{2} \mathrm{~m}}{50}} \operatorname{erfc}\left(\rho \sqrt{\frac{m}{50}}\right)\right)-\frac{1}{\rho \sqrt{50}} \sum_{j=1}^{m-1} \Psi_{j}\left(\frac{2 S_{\mathrm{m}-\mathrm{j}+1}}{\sqrt{\pi}}+\frac{\sqrt{50} R_{m-j+1}}{\rho}\right)-\frac{\mathrm{e}^{\phi_{\mathrm{m}}}}{50} \sum_{j=1}^{m-1} \Psi_{\mathrm{j}}\right]}{1+\omega \mathrm{e}^{-\alpha \phi_{\mathrm{m}}}\left[\frac{1}{\rho \sqrt{50}}\left(\frac{2}{\sqrt{\pi}}+\frac{\sqrt{50} R_{1}}{\rho}\right)+\frac{\mathrm{e}^{\phi_{\mathrm{m}}}}{50}\right]} \text { for reaction II }
$$

$$
\Psi_{\mathrm{m}}=\frac{\omega \mathrm{e}^{-\alpha \phi_{\mathrm{m}}}\left[\frac{1}{\rho}\left(1-\mathrm{e}^{\frac{\rho^{2} \mathrm{~m}}{50}} \operatorname{erfc}\left(\rho \sqrt{\frac{m}{50}}\right)\right)-\frac{1}{\rho \sqrt{50}} \sum_{j=1}^{m-1} \Psi_{j}\left(\frac{2 S_{\mathrm{m}-\mathrm{j}+1}}{\sqrt{\pi}}+\frac{\sqrt{50} R_{\mathrm{m}-\mathrm{j}+1}}{\rho}\right)-\frac{\mathrm{e}^{\phi_{\mathrm{m}}}}{50} \mathrm{e}^{-\frac{\zeta}{25}\left(\Psi_{m}+\sum_{j=1}^{m 1} \Psi_{\mathrm{j}}\right)} \sum_{j=1}^{m-1} \Psi_{\mathrm{j}}\right]}{1+\omega \mathrm{e}^{-\alpha \phi_{\mathrm{m}}}\left[\frac{1}{\rho \sqrt{50}}\left(\frac{2}{\sqrt{\pi}}+\frac{\sqrt{50} R_{1}}{\rho}\right)+\frac{\mathrm{e}^{\phi_{\mathrm{m}}}}{50}\right]}
$$

Here, $\Psi=I /\left(n F A c_{\mathrm{M}^{\mathrm{n}}}^{*}(D f)^{1 / 2}\right)$ and is dimensionless current, where $f$ is the frequency of the potential modulation.

$S_{\mathrm{m}}=(\mathrm{m})^{1 / 2}-(m-1)^{1 / 2}$ and $R_{\mathrm{m}}=\exp \left(\left(\rho^{2} m\right) / 50\right) \operatorname{erfc}(\rho(m)$ $\left.50)^{1 / 2}\right)-\exp \left[\left(\rho^{2}(m-1)\right) / 50\right] \operatorname{erfc}(\rho(m-1) / 50)^{1 / 2}$ and are numerical integration factors. $\lambda=k_{s} /(D f)^{1 / 2}$ and $\gamma=r_{s}(D / f)^{1 / 2}$ and are electrode kinetic and diffusion parameters, respectively, for Reaction I. $\omega=k_{\text {sur }} / f$ and $\rho=(1 / \beta)(D / f)^{1 / 2}$ and are dimensionless electrode kinetic and adsorption parameter, respectively, valid for Reactions II and III, and $\zeta=a(D /$ $f)^{1 / 2}\left(c_{\mathrm{M}^{\mathrm{n}}}^{*} / \Gamma_{\max }\right)$ and is the dimensionless interaction parameter for Reaction III. For numerical integration, each potential pulse of the SW potential modulation is divided into 25 time increments. The serial number of time increments is designated with $m$. From a mathematical aspect (17) is an implicit equation, which must be solved numerically. In the current study, all mathematical solutions are obtained using MATHCAD software package. ${ }^{29}$

\section{EXPERIMENTAL SECTION}

Apparatus. Anodic stripping voltammetric measurements were performed with the modular electrochemical workstation (Autolab, Eco Chemie, Utrecht, The Netherlands) equipped with PGSTAT 12 and driven by GPES software (Eco Chemie). A three-electrode configuration was employed, consisting of a bismuth film modified glassy carbon electrode $(d=2 \mathrm{~mm}), \mathrm{Ag} /$ $\mathrm{AgCl}(\mathrm{KCl}$ std.), and a platinum wire as working, reference, and counter electrodes, respectively. All electrochemical experiments were carried out in a one-compartment voltammetric cell $(20 \mathrm{~mL})$ at conditioned room temperature $\left(23 \pm 1^{\circ} \mathrm{C}\right)$.
Reagents. Standard stock solutions of bismuth(III), cadmium(II), lead(II), and zinc(II) $\left(1 \mathrm{~g} \mathrm{~L}^{-1}\right.$, atomic absorption standard solution) were obtained from Aldrich and diluted as required. A $0.1 \mathrm{M}$ sodium acetate buffer solution ( $\mathrm{pH} 4.5$ ) served as the supporting electrolyte. Water used to prepare all solutions throughout the work was first deionized and then further purified via an Elix 10/Milli-Q Gradient unit (Millipore, Bedford, MA).

Procedure. Anodic stripping voltammetric measurements were performed by the in situ deposition of bismuth film and target metals in the presence of dissolved oxygen. Prior to its use, the glassy carbon disk electrode was polished with a 0.05 $\mu \mathrm{m}$ alumina slurry on a polishing pad. The three electrodes were immersed into a $20 \mathrm{~mL}$ electrochemical cell, containing $0.1 \mathrm{M}$ acetate buffer solution ( $\mathrm{pH} 4.5$ ) and $500 \mu \mathrm{g} \mathrm{L}^{-1}$ of bismuth ions. The deposition potential of $-1.4 \mathrm{~V}$ was applied to the glassy carbon working electrode, while the solution was stirred. Following the preconcentration step (usually $120 \mathrm{~s}$ ), the stirring was stopped, and after an equilibration period of $10 \mathrm{~s}$, the voltammogram was recorded by applying a positive-going square-wave $(\mathrm{SW})$ potential modulation with a potential step of $2 \mathrm{mV}$. Aliquots of the target metal ion standard solution were introduced after recording the background voltammogram. Prior to the next cycle, a $30 \mathrm{~s}$ conditioning step at $+0.3 \mathrm{~V}$ under stirring conditions was used to remove the target metals and the bismuth film. 


\section{RESULTS AND DISCUSSION}

Simple Anodic Stripping Mechanism (Reaction I). Typical response of Reaction I is depicted on panel A of Figure 1. The forward component of the SW response reflects the
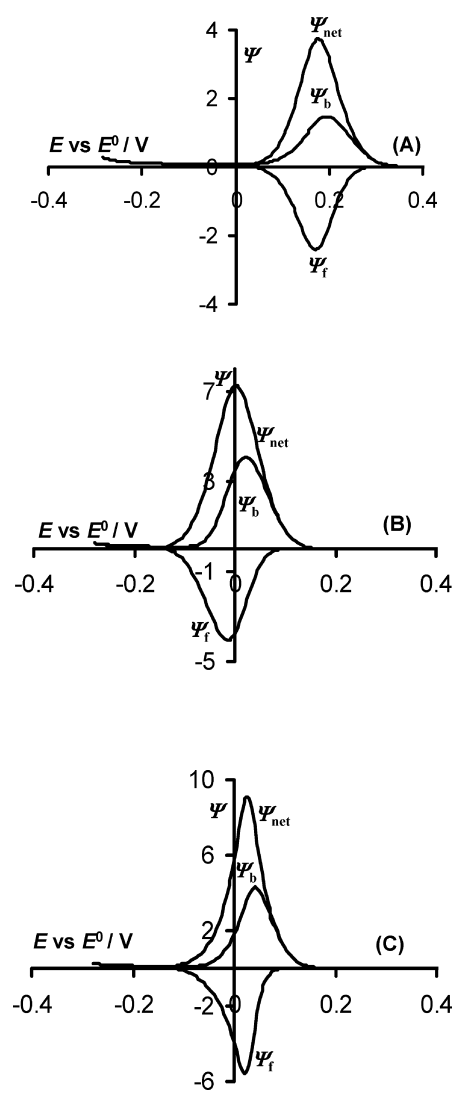

Figure 1. Typical SW voltammograms calculated for (A) simple anodic stripping Reaction I, (B) adsorption coupled Reaction II, and (C) interaction complicated Reaction III. The standard rate constant is $k_{\mathrm{s}}=1 \mathrm{~cm} \mathrm{~s}^{-1}$ for (A) and $k_{\text {sur }}=50 \mathrm{~s}^{-1}$ for both (B) and (C). The adsorption constant is $\beta=0.01 \mathrm{~cm}$ (for $\mathrm{B}$ and $\mathrm{C}$ ) and the Frumkin interaction parameter is $a=3, \Gamma_{\max }=10^{-10} \mathrm{~mol} \mathrm{~cm}^{-2}$, and $c^{*}{ }_{\mathrm{M}}=1 \times$ $10^{-8} \mathrm{~mol} \mathrm{~cm}^{-3}$ (for $\mathrm{C}$ ). The other conditions of the simulations are: $\alpha$ $=0.5, n=1, D=5 \times 10^{-6} \mathrm{~cm}^{2} \mathrm{~s}^{-1}, T=298.15 \mathrm{~K}, f=50 \mathrm{~Hz}, E_{\mathrm{sw}}=50$ $\mathrm{mV}$ (amplitude), $\Delta E=5 \mathrm{mV}$ (step potential), and $E_{\mathrm{s}}=-0.3 \mathrm{~V}_{\mathrm{vs}} \mathrm{E}_{\mathrm{c}}^{\ominus^{\prime}}$ (starting potential).

anodic stripping process of the metal analyte deposit from the BiFE, whereas the backward component represents the subsequent reduction of the metal ions in the course of the reverse cathodic potential pulses of the SW potential modulation. The anodic component has a smaller half-peak width $\left(\Delta E_{\mathrm{p} / 2}\right)$, as typical for electrode processes of an immobilized reactant, whereas the backward component is wider, due to the diffusion mass transfer control of metal ions. Potential separation between the forward and backward components and, hence, the half-peak width of the net SW peak depend predominantly on the electrochemical reversibility and the product $n E_{s w}$, where $E_{s w}$ is the amplitude of the potential modulation and $n$ is the number of exchanged electrons. Note that the forward (anodic) stripping component of the response is located at more negative potential relative to the backward (cathodic) one, which is the consequence of the specific presentation of the voltammetric data in square-wave voltammetry (SWV). ${ }^{19}$
The voltammetric response of the mechanism I is predominantly controlled by the dimensionless electrode kinetic parameters $\lambda=k_{\mathrm{s}} /(D f)^{1 / 2}$ and the diffusion parameter $\gamma=r_{s}(D / f)^{1 / 2}$. Obviously, $\lambda$ combines the rate of the electron exchange (standard rate constant, $k_{\mathrm{s}}$ ), the rate of mass transfer $(D)$, and the time window of the voltammetric experiment $(f)$, whereas $\gamma$ represents only the effect of mass transfer. However, the electrochemical reversibility is solely controlled by the complex, implicit parameter $\xi=\lambda(\gamma)^{1 / 2}$. In terms of the real parameters, $\xi$ is defined as $\xi=k_{s} D^{-1 / 4} f^{-3 / 4} r_{s}^{1 / 2} .^{30}$ The electrode reaction is electrochemically reversible when $k_{\mathrm{s}}>$ $300 D^{1 / 4} f^{3 / 4} r_{s}^{-1 / 2}$, while, for $k_{\mathrm{s}}<5 \times 10^{-3} D^{1 / 4} f^{3 / 4} r_{\mathrm{s}}^{-1 / 2}$, the reaction is irreversible. Within these limits, the reaction is quasireversible.

Studying the single electrode reaction, characterized with particular value of $k_{s}$ and $D$, the electrochemical reversibility parameter $\xi$ can be altered by varying the SW frequency. Figure 2 depicts the effect of the frequency on the dimensionless

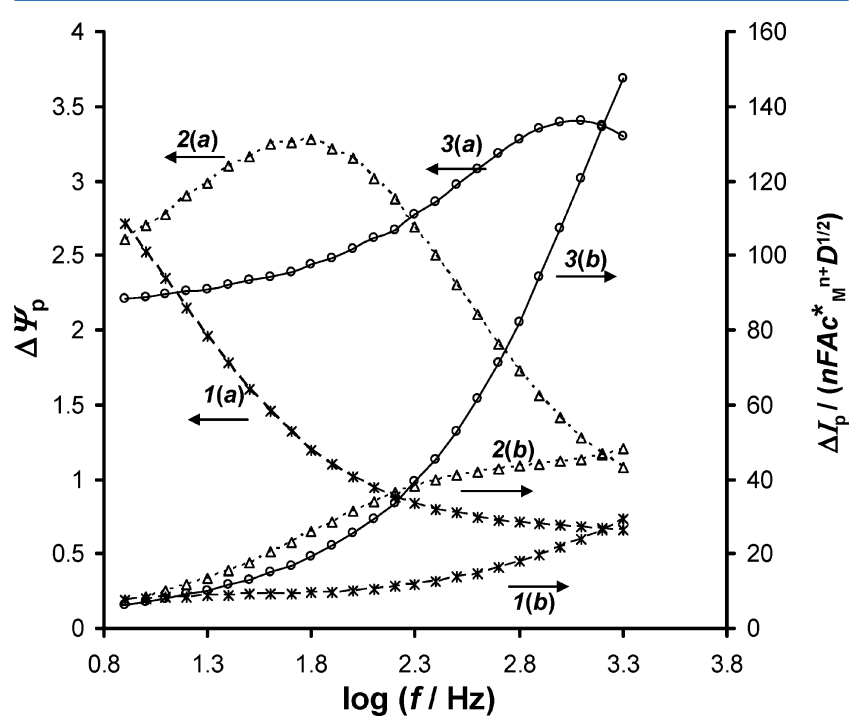

Figure 2. Simple anodic stripping Reaction I. Dependence of the dimensionless net peak current (left ordinate, curves designated with (a)) and normalized real net peak current (right ordinate, curves designated with (b)) on the logarithm of the SW frequency. The standard rate constant is $k_{\mathrm{s}} /\left(\mathrm{cm} \mathrm{s}^{-1}\right)=0.1$ (1); 1 (2) and 10 (3). Other conditions are the same as for Figure 1.

$\left(\Delta \Psi_{\mathrm{p}}\right)$ and normalized real $\left(\Delta I_{\mathrm{p}}\right)$ net peak currents for different values of $k_{s}$. Generally speaking, the real net peak current $\left(\Delta I_{\mathrm{p}}\right)$ increases nonlinearly with $f$; the degree of enhancement, i.e., $\partial \Delta I_{\mathrm{p}} / \partial(\log f)$ depends on the frequency interval as well as the standard rate constant. Dimensionless net peak current $\left(\Delta \Psi_{\mathrm{p}}\right)$ depends also nonlinearly on $f$; however, within the quasireversible kinetic region, the dependence exhibits a pronounced maximum, which is the well-know feature of stripping processes called "quasireversible maximum". ${ }^{31}$ In agreement with previous findings, the position of the quasireversible maximum depends on particular value of $k_{s}$, revealing that it can be exploited for estimation of the latter kinetic parameter $\left(k_{\mathrm{s}}\right)$ in a simple procedure elaborated in detail in previous studies. ${ }^{30,31}$ In the reversible kinetic region, the real net peak current depends linearly on the square-root of the frequency, while the net peak potential shifts linearly with $\log (f)$ toward more positive potentials. 
Alongside to the frequency, the SW amplitude is the second powerful instrumental tool to study the electrode reaction for the purpose of the electrode kinetic measurements, as well as optimization of the response for analytical application. For kinetic measurements, i.e., estimation of $k_{\mathrm{s}}$, it is particularly useful to analyze the peak potential separation between the anodic (forward) and cathodic (backward) components of the response by altering $E_{\mathrm{sw}}$, while keeping the frequency constant. This methodology will be illustrated in the forthcoming section devoted to the adsorption complicated electrode mechanism II. From an analytical point of view, the amplitude is also particularly important, as it affects both the peak current and half-peak width $\left(\Delta E_{\mathrm{p} / 2}\right)$ of the net $S W$ response. The analytically optimal net SW peak would be characterized with the maximal value of the ratio $\Delta \Psi_{\mathrm{p}} / \Delta E_{\mathrm{p} / 2}$. Figure 3 shows the

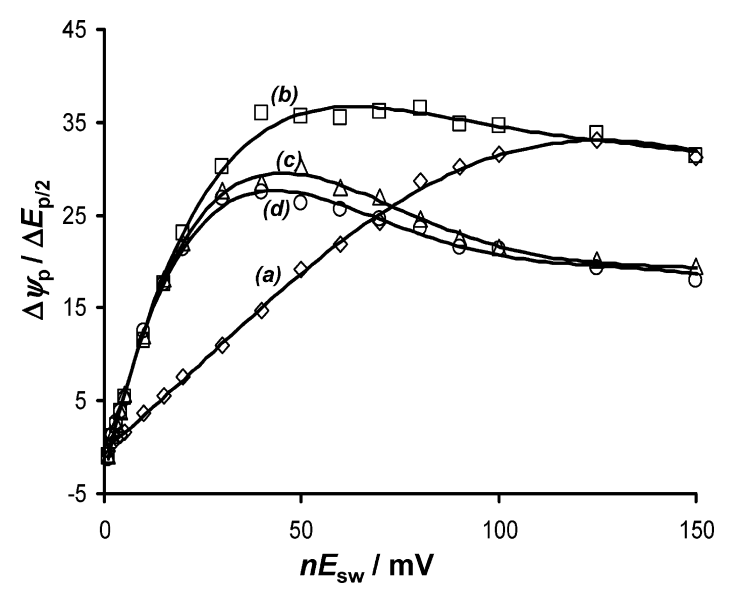

Figure 3. Simple anodic stripping Reaction I. Dependence of the ratio $\Delta \Psi_{\mathrm{p}} / \Delta E_{\mathrm{p} / 2}$ on the $S W$ amplitude for different reversibility of the electrode reaction $\left(\Delta E_{\mathrm{p} / 2}\right.$ is the half-peak width). The standard rate constant is $k_{\mathrm{s}} /\left(\mathrm{cm} \mathrm{s}^{-1}\right)=0.1(\mathrm{a}) ; 1$ (b); 5 (c), and $10(\mathrm{~d})$. The frequency and the step potential are $f=25 \mathrm{~Hz}$ and $\Delta E=5 \mathrm{mV}$. Other conditions are the same as for Figure 1.

variation of the ratio $\Delta \Psi_{\mathrm{p}} / \Delta E_{\mathrm{p} / 2}$ with the product $n E_{\mathrm{sw}}$ for different electrochemical reversibilities of the electrode reactions. When the reaction is slow and electrochemically irreversible (curve a in Figure 3), a relatively large amplitude is required, i.e., $n E_{\mathrm{sw}}>100 \mathrm{mV}$, whereas for quasireversible electrode reactions, the product $n E_{\mathrm{sw}} \approx 50 \mathrm{mV}$ provides optimal conditions for analytical application (curves $b-d$ in Figure 3).

Anodic Stripping Mechanism Coupled with Adsorption (Reaction II). As shown in panel B of Figure 1, the adsorption of the metal ion analyte on the BiFE affects significantly the overall voltammetric behavior, producing a specific shape of the stripping voltammetric curves. Contrary to the mechanism I, in the present case, both components of the SW response are sharp peaks, as is typical for the surface electrode process of an immobilized redox couple. ${ }^{32}$ When adsorption strength is significant, both components have almost identical height, resulting in a large and intensive net SW peak, which is favorable for analytical application.

As implied by eq 16 , the dimensionless response is mainly governed by the electrode kinetic parameter $\omega=k_{\text {sur }} / f$ and the adsorption parameter $\rho=(1 / \beta)(D / f)^{1 / 2}$. The electrochemical reversibility is solely determined by $\omega$, the reaction being reversible for $k_{\text {sur }}>100 f$ and irreversible for $k_{\text {sur }}<0.01 f$. Within these limits, the electrode reaction is quasireversible, being characterized with a well-developed, sharp quasireversible maximum. The adsorption parameter combines the strength of adsorption $(\beta)$ with the diffusion rate and the time window of the voltammetric experiment. The strength of adsorption affects the net peak current in a complex manner, depending mainly on the electrochemical reversibility of the electrode reaction. For low electrochemical reversibility $(\omega<1)$, the stronger the adsorption, the higher is the net SW peak. On the contrary, for relatively high electrochemical reversibility $(\omega>$ $5)$, the intensity of the response decreases by increasing the adsorption strength, due to the specific chronoamperometric properties of surface electrode reactions, where the current severely diminishes with time in the course of the potential pulses. It is important to note that the net peak potential and half-peak width are virtually insensitive to the adsorption of the metal ion analytes, which simplifies the analytical application.

Studying a single electrode reaction, the adsorption constant can be altered by adjusting the chemical composition and concentration of the electrolyte solution. In addition, the adsorption parameter can be altered by adjusting the frequency of the potential modulation. However, one should be aware that variation of the frequency will affect simultaneously both parameters $\omega$ and $\rho$; thus, the generalization of the frequency effect is not straightforward. For this mechanism, the role of the SW amplitude is of particular importance, as it affects strongly the potential separation between the forward and backward components of the response. Figure 4 reveals that the peak

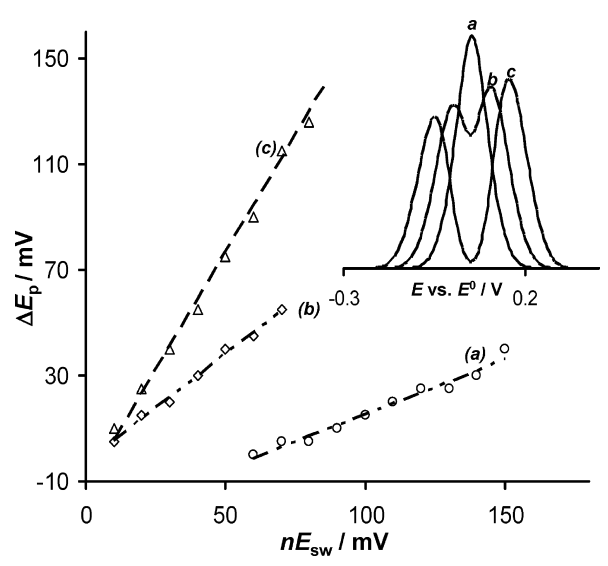

Figure 4. Adsorption coupled anodic stripping Reaction II. The dependence of the peak potential separation $\left(\Delta E_{\mathrm{p}}\right)$ on the SW amplitude $\left(n E_{\text {sw }}\right)$. The standard rate constant is $k_{\text {sur }} / \mathrm{s}^{-1}=1$ (a), 10 (b), and 50 (c). The adsorption constant is $\beta=0.1 \mathrm{~cm}$, and the frequency is $f=10 \mathrm{~Hz}$. Other conditions are the same as for Figure 1 .

potential separation is a linear function of the product $n E_{\mathrm{sw}}$. The slope and the intercept of the lines are sensitive to the standard rate constant, implying that this sort of analysis is particularly appealing for kinetic measurements. Such methodology is advantageous, as it requires alteration of the potential amplitude only, while keeping the frequency, i.e., the scan rate ( $\nu=f \Delta E$, where $\Delta E$ is the step potential) at a constant value, which is the unique ability of the SWV. However, from an analytical standpoint, the enlargement of the peak potential separation is unfavorable, as it increases the half-peak width of the net peak; moreover, at sufficiently large amplitudes the net SW peak could be split, as shown in the inset of Figure 4.

Anodic Stripping Mechanism with Interactions (Reaction III). In addition to the parameters $\omega$ and $\rho$, the response 
of electrode Reaction III depends on the dimensionless parameter $\zeta=a(D / f)^{1 / 2} \cdot\left(\mathrm{c}_{\mathrm{M}}^{*}{ }^{\mathrm{n}}\right) /\left(\Gamma_{\max }\right)$, where $a$ is the Frumkin interaction parameter. A typical SW voltammetric response, compared to the responses of the two previous mechanisms, is depicted in Figure 1C. Attractive and repulsive lateral interactions are associated with $a>0$ and $a<0$, respectively. The overall interaction effect depends on the fractional coverage of the electrode, $\theta=\left(\Gamma_{\mathrm{M}}\right) /\left(\Gamma_{\max }\right)$; hence, all parameters affecting the surface concentration of the metal analyte deposit (accumulation time, $D, f$ ) will affect the degree of interactions. As shown in Figure 5B,C, the morphology of
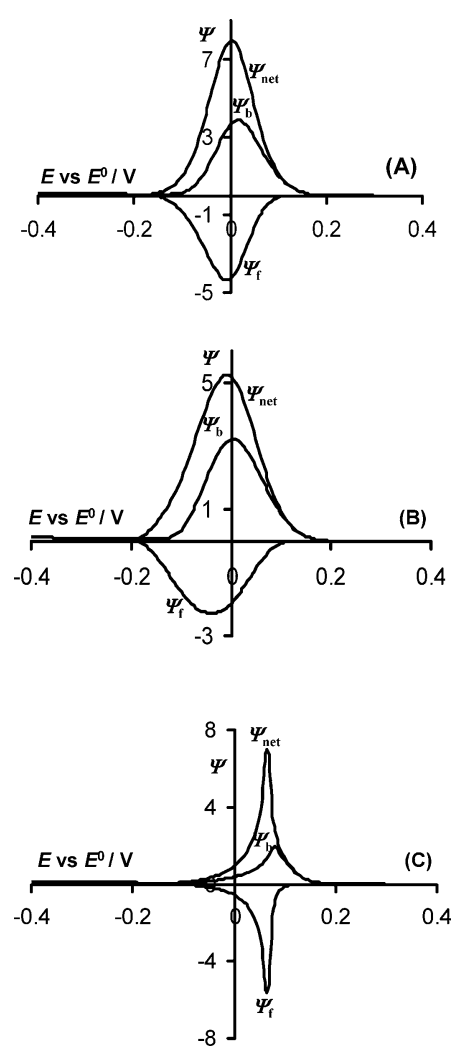

Figure 5. Typical SW voltammograms calculated for electrode reaction involving interactions between deposited metal particles (Reaction III). The Frumkin interaction parameter is $a=0$ (A), -2 (B), and 2 (C). The other parameters are $\beta=0.1 \mathrm{~cm}, k_{\mathrm{sur}}=5 \mathrm{~s}^{-1}, f=50 \mathrm{~Hz}$, and

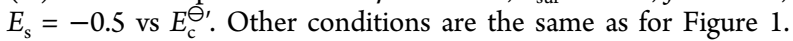

the response is markedly susceptible on the type of interactions compared to the interaction-free case (Figure 5A). For repulsive forces (Figure 5B), all components of the response are enlarged in their half-peak width and shifted toward less positive potentials. As expected, the anodic stripping process is energetically facilitated by the repulsive forces. On the contrary, under attractive forces (Figure 5C), additional energy is required to account for the interactions, causing a shift of the response toward more positive potentials. Moreover, voltammetric curves are particularly sharp, which provides a simple diagnostic criterion to make a distinction between the type of interactions involved.

As follows from the definition of the dimensionless interaction parameter $\xi$, for a given value of the Frumkin interaction parameter $a$, the degree of interactions will enlarge by increasing the bulk concentration of the metal ion studied, while the frequency of the potential modulation will exhibit an opposite effect. Therefore, the potential shift of the anodic stripping voltammetric response by increasing the concentration of the ion will serve as a simple diagnostic criterion for the presence and the type of interactions, which is a unique feature of this mechanism.

Experimental Results. From the relation between the real and dimensionless current, $\Psi=I /\left(n F A c_{\mathrm{M}^{\mathrm{n+}}}^{\mathrm{n}}(D F)^{1 / 2}\right)$, it is obvious that, for particular electrode reaction and a given set of experimental conditions, voltammetric features are determined by properties of the function $\Psi$. Figure 6 depicts the anodic

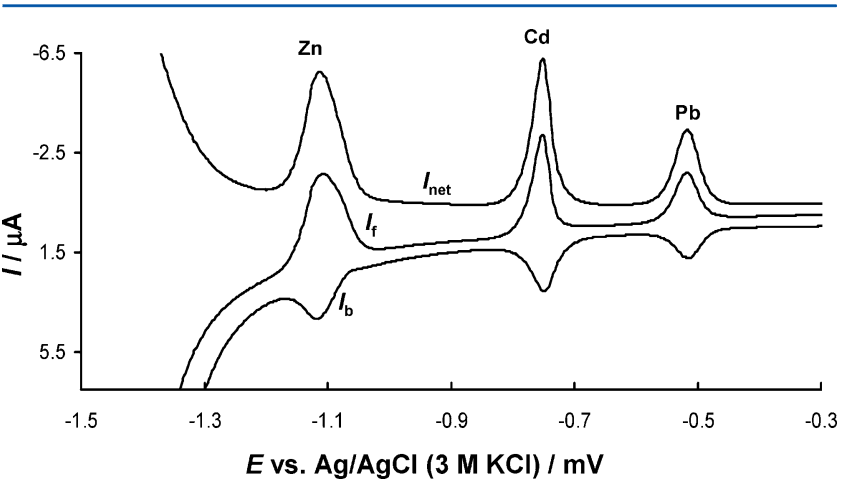

Figure 6. Typical anodic stripping SW voltammetric response of $50 \mu \mathrm{g}$ $\mathrm{L}^{-1} \mathrm{Zn}^{2+}(\mathrm{aq}), \mathrm{Cd}^{2+}(\mathrm{aq})$, and $\mathrm{Pb}^{2+}(\mathrm{aq})$ at $\mathrm{BiFE}$ recorded in an acetate buffer at $\mathrm{pH}$ 4.4. The Bi-film is in situ plated on the surface of the glassy carbon electrode from solution containing $1 \mathrm{mg} \mathrm{L}^{-1} \mathrm{Bi}^{3+}$ (aq). The deposition was conducted at $-1.4 \mathrm{~V}$ for $120 \mathrm{~s}$ with stirring of the solution, followed by $10 \mathrm{~s}$ equilibrium time. The SW parameters are $f=$ $20 \mathrm{~Hz}, E_{\mathrm{sw}}=20 \mathrm{mV}$, and $\Delta E=2 \mathrm{mV}$.

stripping SW voltammetric response at BiFE of zinc(II), cadmium(II), and lead(II), all analytes being simultaneously present in the same electrolyte solution at an equal mass concentration, the molar concentration ratio being $c(\mathrm{Zn}(\mathrm{II})) /$ $c(\mathrm{Cd}(\mathrm{II})) / c(\mathrm{~Pb}(\mathrm{II}))=3.2: 1.8: 1$. Although $n=2$ for all electrode reactions, as well as $D$ is quite comparable for all metal ions, their anodic stripping responses are significantly different in many respects, implying different electrode mechanisms associated with different $\Psi$ functions. The net peak current ratio is $\Delta I_{\mathrm{p}}(\mathrm{Zn}(\mathrm{II})) / \Delta I_{\mathrm{p}}(\mathrm{Cd}(\mathrm{II})) / \Delta I_{\mathrm{p}}(\mathrm{Pb}(\mathrm{II}))=$ 1.6:1.9:1, which does not correspond to the molar concentration ratio. From all three analytes, cadmium gives raise to the narrowest $\left(\Delta E_{\mathrm{p} / 2}=34 \mathrm{mV}\right)$ net $\mathrm{SW}$ peak. Particular attention should be given to the properties of the forward (anodic stripping) and backward (cathodic) components of the responses, as they reveal important characteristics of a particular electrode mechanism. For zinc, the forward component is located at more positive potentials compared to the backward one, with a peak potential separation of $\Delta E=6$ $\mathrm{mV}$. Interestingly, the forward stripping component is attributed with a half-peak width of $\Delta E_{\mathrm{p} / 2}=73 \mathrm{mV}$, being broader than the backward one $\left(\Delta E_{\mathrm{p} / 2}=52 \mathrm{mV}\right)$. The peak current ratio between the forward and backward components is $I_{\mathrm{p}, \mathrm{f}} / I_{\mathrm{p}, \mathrm{b}}=2.68$, which is the highest value for all three analytes. The response of cadmium is significantly different, with a forward component positioned at slightly more negative potential compared to the backward one, while the peak potential separation is only $2 \mathrm{mV}$. Both forward and backward components are narrow peaks with half-peak widths of 32 and $44 \mathrm{mV}$, respectively. The peak current ratio is $I_{\mathrm{p}, \mathrm{f}} / I_{\mathrm{p}, \mathrm{b}}=1.99$, being smaller than for zinc. Similar to cadmium, the response of lead consists of symmetric forward and backward components, 
with a peak potential separation of $2 \mathrm{mV}$. However, in comparison to cadmium, all components of lead response are broader $\left(\Delta E_{\mathrm{p} / 2}=44 \mathrm{mV}\right.$, net $S W$ peak $)$, while the peak current ratio is smaller $\left(I_{\mathrm{p}, \mathrm{f}} / I_{\mathrm{p}, \mathrm{b}}=1.77\right)$.

The morphological analysis of the single voltammogram reveals that no electrode reaction proceeds according to the simple anodic stripping mechanism (Reaction I). The morphology of the voltammograms of cadmium and lead imply strongly that the electrode mechanism is coupled with adsorption of the metal ions (Reaction II). However, both voltammograms are narrower than expected for Reaction II, indicating that the electrode reactions are complicated by attractive forces within the metal deposit (Reaction III). This is particularly emphasized for cadmium, which is attributed with the narrowest net SW peak. For the case of lead, the interactions are less significant, however, the adsorption of $\mathrm{Pb}^{2+}$ (ads) at $\mathrm{BiFE}$ appears stronger. The peak current ratio shows that, following the anodic stripping of the metal analyte deposit, the majority of metal ions remain immobilized on the electrode surface. The simulations with the Reaction II clearly show that the peak current ratio $I_{\mathrm{p}, \mathrm{f}} / I_{\mathrm{p}, \mathrm{b}}$ decreases by increasing the strength of adsorption.

The morphology of the zinc response does not imply significant adsorption of $\mathrm{Zn}^{2+}(\mathrm{aq})$ ions, as the backward component exhibits properties of a diffusion controlled process, as predicted by Reaction I. However, the complexity of the electrode mechanism is reflected mainly through the features of the forward stripping component, which is unusually broad. The latter might be a consequence of overlapping of two separate stripping peaks, due to multilayer metal deposit, as observed in the study. ${ }^{33}$ An alternative explanation can be found with the aid of the model Reaction III, assuming repulsive interactions within the metal deposit. The latter mechanism also explains the observed shift of the forward stripping peak (and thus the net peak) toward more negative potentials by increasing the bulk concentration of $\mathrm{Zn}^{2+}(\mathrm{aq})$ ions. We recall to the definition of $\zeta=a(D / f)^{1 / 2}\left(\mathrm{c}_{\mathrm{M}^{\mathrm{n}}}^{*_{\mathrm{n}}} / \Gamma_{\max }\right)$, showing that the degree of interactions is proportional to the bulk concentration of metal ions. The same potential shift of the anodic stripping response of zinc was observed in the study of Kefala et al. ${ }^{34}$ On the contrary, increasing $\mathrm{Cd}^{2+}(\mathrm{aq})$ and $\mathrm{Pb}^{2+}(\mathrm{aq})$ concentrations causes a slight shift of the voltammetric stripping peaks to positive potential direction, being in agreement with the assumption that the electrode mechanism involves attractive interactions. ${ }^{34}$

For all three ions, an increase of the frequency causes a nonlinear increase of the net peak current, which is a general characteristic of all three model reactions. Over the frequency interval $8 \mathrm{~Hz} \leq f \leq 120 \mathrm{~Hz}$, the frequency normalized net peak current, $\Delta I_{\mathrm{p}} f^{-0.5}$ does not exhibit the quasireversible maximum, indicating that within this frequency range all electrode reactions are attributed with a high degree of electrochemical reversibility.

In agreement with the theoretical predictions, the SW amplitude exhibits strong influence on the peak current and the half-peak width of the net response of all analytes. The ratio $\Delta I_{\mathrm{p}} / \Delta E_{\mathrm{p} / 2}$ exhibits a maximal value for an amplitude value close to $50 \mathrm{mV}$, which is optimal for analytical applications for all three analytes, being in agreement with the theoretical predictions. Besides, the peak potential separation between the forward and backward components of the SW responses for all three analytes depends linearly on the SW amplitude as shown in Figure 7. The slopes of the lines are different, as the

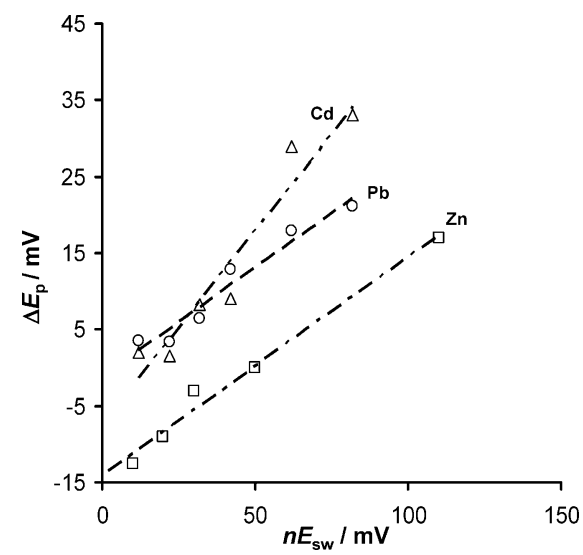

Figure 7. Dependence of the peak potential separation $\left(\Delta E_{\mathrm{p}}\right)$ on the SW amplitude $\left(n E_{\mathrm{sw}}\right)$ for all three analytes. The experimental conditions are the same as for Figure 6.

electrode reactions are attributed with different electrochemical reversibilities. We finally note that comparison of these data presented in Figure 7 for all three analytes is not straightforward, as all electrode mechanisms are significantly different.

\section{CONCLUSIONS}

In this paper, it has been demonstrated that the proposed theoretical model combined with numerical simulations under square-wave voltammetry conditions can provide a basis for qualitative characterization of anodic stripping mechanisms while using BiFE as the most attractive ASV substrate in recent times. The proposed theoretical consideration is not restricted to the BiFEs only. Rather, it can be applied to any anodic stripping process where the analyte is electrochemically deposited onto a suitable substrate surface, forming a film (in an ideal case a monolayer), which is uniform with respect to the energy required for its anodic stripping. Each electrode mechanism considered is represented with a single recurrent formula that can be easily implemented in various commercially available spread-sheet or mathematical software packages to predict the outcome of a voltammetric experiment, without requiring specialized electrochemical software for simulations. Presented formulas reveal critical dimensionless parameters that govern intrinsic voltammetric features, which elucidate the operative electrode mechanism and enable diagnostic criteria to be established. With a careful comparison of the simulated and experimental data, it is possible to differentiate the operative electrode mechanism, even by analyzing the morphology of a single voltammogram. On the basis of the presented theoretical data, as well as from the previous knowledge of related electrode mechanisms, two strategies for kinetic characterization of anodic stripping processes are derived: (i) the first one requires inspection of the net SW peak current as a function of the SW frequency (i.e., the scan rate $v=f \Delta E$ ), exploiting the feature known as a "quasireversible maximum", and (ii) the second approach is based on measuring the peak potential separation between forward and backward components of the SW response as a function of the SW amplitude. The exclusivity of the latter approach stems from the fact that it enables kinetic measurements at a constant frequency (i.e., constant scan rate), which appears to be the unique ability of square-wave voltammetry. Finally, the presence of significant lateral interactions between the metal analyte particles can be 
voltammetrically detected and characterized by analyzing the morphology of the voltammetric response, as well as by studying the variation of net SW peak potential variation with the metal analyte concentration in the electrolyte solution. In this study, the ability of the proposed SWV diagnostic protocol to qualitatively characterize anodic stripping mechanisms by determining the corresponding kinetic parameters has been practically demonstrated using $\mathrm{Zn}(\mathrm{II}), \mathrm{Cd}(\mathrm{II})$, and $\mathrm{Pb}(\mathrm{II})$ as model trace metal analytes while the popular bismuth film in situ deposited onto a glassy carbon electrode served as a model ASV substrate.

\section{AUTHOR INFORMATION}

\section{Corresponding Author}

*E-mail: bogorevc@ki.si.

\section{Notes}

The authors declare no competing financial interest.

\section{ACKNOWLEDGMENTS}

The authors acknowledge the support of the Slovenian Research Agency (ARRS) and the Ministry of Education and Science of the Republic of Macedonia (bilateral project No. BIMK/10-11-005). B.O. and S.B.H. thank the ARRS for financial support (Program No. P1-0034-0104). V.M. and R.G. also acknowledge the support of the $\mathrm{AvH}$ foundation through the Research Group Linkage Programme (3.4-Fokoop-DEU/ $1128670)$ as well as the support of DAAD foundation through multilateral MatCatNet project.

\section{REFERENCES}

(1) Wang, J. Analytical Electrochemistry, 3rd ed.; Wiley-VCH: Hoboken, NJ, 2006

(2) Williams, D. G.; Johnson, D. C. Anal. Chem. 1992, 64, 17851789 .

(3) Brand, M.; Eshkenazi, I.; Kirowa-Eisner, E. Anal. Chem. 1997, 69, $4660-4664$

(4) Nolan, M. A.; Kounaves, S. P. Anal. Chem. 1999, 71, 3567-3573.

(5) Wang, J.; Lu, J.; Hocevar, S. B.; Farias, P. A. M.; Ogorevc, B. Anal. Chem. 2000, 72, 3218-3222.

(6) Hocevar, S. B.; Svancara, I.; Ogorevc, B.; Vytras, K. Anal. Chem. 2007, 79, 8639-8643.

(7) Guzsványa, V.; Nakajima, H.; Soh, N.; Nakanob, K.; Imato, T. Anal. Chim. Acta 2010, 658, 12-17.

(8) Urbanová, V.; Vytrăs, K.; Kuhn, A. Electrochem. Commun. 2010, $12,114-117$.

(9) Wang, J.; Lu, J.; Kirgöz, Ü. A.; Hocevar, S. B.; Ogorevc, B. Anal. Chim. Acta 2001, 434, 29-34.

(10) Hutton, E. A.; Hocevar, S. B.; Ogorevc, B. Anal. Chim. Acta 2005, 537, 285-292.

(11) Serrano, N.; Díaz-Cruz, J. M.; Ariño, C.; Esteban, M. Anal. Bioanal. Chem. 2010, 396, 1365-1369.

(12) Wang, J.; Lu, J. Electrochem. Commun. 2000, 2, 390-393.

(13) Hutton, E. A.; Elteren van, J. T.; Ogorevc, B.; Smyth, M. R. Talanta 2004, 63, 849-855.

(14) Królicka, A.; Bobrowski, A. Electrochem. Commun. 2004, 6, 99104.

(15) Hutton, E. A.; Ogorevc, B.; Hocevar, S. B.; Weldon, F.; Smyth, M. R.; Wang, J. Electrochem. Commun. 2001, 3, 707-711.

(16) Frena, M.; Campestrini, I.; De Braga, O. C.; Spinelli, A. Electrochim. Acta 2011, 56, 4678-4684.

(17) Segura, R.; Pradena, M.; Pinto, D.; Godoy, F.; Nagles, E.; Arancibia, V. Talanta 2011, 85, 2316-2319.

(18) Saturno, J.; Valera, D.; Carrero, H.; Fernandez, L. Sens. Actuators B 2011, 159, 92-96.
(19) Mirceski, V.; Komorsky-Lovric, S.; Lovric, M. Square-Wave Voltammetry: Theory and Applications; Scholz, F., Ed.; Springer: Heidelberg, 2007.

(20) Kounaves, S. P.; O’Dea, J. J.; Chandrasekhar, P.; Osteryoung, J. Anal. Chem. 1986, 58, 3199-3202.

(21) Kounaves, S. P.; O’Dea, J. J.; Chandrasekhar, P.; Osteryoung, J. Anal. Chem. 1987, 59, 386-389.

(22) Penczek, M.; Stojek, Z. J. Electroanal. Chem. 1986, 213, 177188.

(23) Mlakar, M.; Lovric, M. Analyst 1990, 115, 45-48.

(24) Ball, J. C.; Compton, R. G. J. Phys. Chem. B 1998, 102, 39673973.

(25) Miles, A. B.; Compton, R. G. J. Electroanal. Chem. 2000, 487, $75-89$.

(26) Lovric, M.; Branica, M. J. Electroanal. Chem. 1987, 226, 239251.

(27) Laviron, E.; Roullier, L. J. Electroanal. Chem. 1980, 115, 65-74.

(28) Nicholson, R. S.; Olmstead, M. L. Electrochemistry: Calculations, Simulation and Instrumentation, Vol. 2; Mattson, J. S., Mark, H. B., MacDonald, H. C., Eds.; Marcel Dekker: New York, 1972; pp 120137.

(29) MATHCAD; MathSoft Inc.: Cambridge, MA, 1998.

(30) Mirceski, V.; Lovric, M. Electroanalysis 1999, 11, 984-989.

(31) Gulaboski, R.; Mirceski, V.; Komorsky-Lovric, S.; Lovric, M. Electroanalysis 2004, 16, 832-842.

(32) Mirceski, V.; Lovric, M. Electroanalysis 1997, 9, 1283-1287.

(33) Serrano, N.; Alberich, A.; Diaz-Cruz, J. M.; Ariño, C.; Esteban, M. Electrochim. Acta 2008, 53, 6616-6622.

(34) Kefala, G.; Economou, A.; Voulgaropoulos, A.; Sofoniou, M. Talanta 2003, 61, 603-610. 